\title{
Feynman's Tree Theorem and Loop-Tree Dualities
}

Isabella Bierenbaum *

Instituto de Física Corpuscular, Universitat de Valencia-Consejo Superior de Investigaciones Científicas, Apartado de Correos 22085, E-46071, Valencia, Spain

E-mail: isabella.bierenbaumeific.uv.es

\section{Stefano Catani}

INFN, Sezione di Firenze and Dipartimento di Fisica, Universita di Firenze, I-50019 Sesto

Fiorentino, Florence, Italy

E-mail: stefano.catani@fi.infn.it

\section{Petros Draggiotis}

Instituto de Física Corpuscular, Universitat de Valencia-Consejo Superior de Investigaciones Científicas, Apartado de Correos 22085, E-46071, Valencia, Spain

E-mail: Petros.Drangiotis@ific.uv.es

\section{German Rodrigo ${ }^{\dagger}$}

Instituto de Física Corpuscular, Universitat de Valencia-Consejo Superior de Investigaciones Científicas, Apartado de Correos 22085, E-46071, Valencia, Spain

E-mail: german.rodrigo@ific.uv.es

We discuss the duality theorem, which provides a relation between loop integrals and phase space integrals. We rederive the duality relation for the one-loop case and extend it to two and higherorder loops. We explicitly show its application to two- and three-loop scalar master integrals and discuss the structure of the occurring cuts.

Light Cone 2010 - LC2010

June 14-18, 2010

Valencia, Spain

*As of October 2010: Institut für Theoretische Teilchenphysik und Kosmologie RWTH Aachen D-52056 Aachen, Germany. E-mail: bierenbaum@physik.rwth-aachen.de

${ }^{\dagger}$ Speaker. 


\section{Introduction}

In recent years, important efforts have been devoted to developing efficient methods for the calculation of multi-leg and multi-loop diagrams, realized, e.g., by unitarity-based methods or by traditional Feynman diagram approaches, [1].

The computation of cross sections at next-to-leading order (NLO) or next-to-next-to-leading order (NNLO) requires the separate evaluation of real and virtual radiative corrections, which are given in the former case by multi-leg tree-level and in the latter by multi-leg loop matrix elements to be integrated over the multi-particle phase-space of the physical process. The loop-tree duality relation at one-loop presented in Ref. [2], as well as other methods relating one-loop and phasespace integrals, $[3,4,5]$, recast the virtual radiative corrections in a form that closely parallels the contribution of the real radiative corrections. The use of this close correspondence is meant to simplify calculations through a direct combination of real and virtual contributions to NLO cross sections. Furthermore, the duality relation has analogies with the Feynman Tree Theorem (FTT), $[6,7]$, but offers the advantage of involving only single cuts of the one-loop Feynman diagrams.

In this talk we will present the equivalence of the FFT and duality theorems at one-loop level and extend it to higher order loops, with the aim of applying it to NLO and NNLO cross sections.

\section{The Duality Theorem at One Loop}

We start by considering a general one-loop $N$-leg diagram, as shown in Fig. 1., which is represented by the scalar integral:

$$
L^{(1)}\left(p_{1}, p_{2}, \ldots, p_{N}\right)=\int_{\ell_{1}} \prod_{i=1}^{N} G_{F}\left(q_{i}\right) .
$$

The four-momenta of the external legs are denoted $p_{i}, i \in\{1,2, \ldots N\}$. All are taken as outgoing and ordered clockwise. We use dimensionally regularized integrals with the number of space-time dimensions equal to $d$, and introduce the following shorthand notation:

$$
\int_{\ell_{i}} \cdots \equiv-i \int \frac{d^{d} \ell_{i}}{(2 \pi)^{d}} \cdots
$$

The FFT and the duality theorem, both depend on the pole structure of the Feynman and the advanced propagators. These are defined as

$$
G_{F}\left(q_{i}\right)=\frac{1}{q_{i}^{2}-m_{i}^{2}+i 0}, \quad G_{A}\left(q_{i}\right)=\frac{1}{q_{i}^{2}-m_{i}^{2}-i 0 q_{i, 0}}
$$

with $q_{i}$ being the $d$-dimensional four momentum, whose energy (time component) is $q_{i, 0}$. The poles of both kinds of propagators in the complex $q_{i, 0}$-plane are placed at:

$$
q_{i, 0}^{F}= \pm \sqrt{\mathbf{q}_{i}^{2}-m_{i}^{2}-i 0}, q_{i, 0}^{A} \simeq \pm \sqrt{\mathbf{q}_{i}^{2}-m_{i}^{2}}+i 0,
$$

respectively. Thus, the pole with positive (negative) energy of the Feynman propagator is slightly displaced below (above) the real axis, while both poles (independent of the sign of energy) of the advanced propagator are slightly displaced above the real axis. Using the elementary identity:

$$
\frac{1}{x \pm i 0}=P V\left(\frac{1}{x}\right) \mp i \pi \delta(x)
$$


where $P V$ denotes the principal value, we find the following relation between the two propagators:

$$
G_{A}\left(q_{i}\right)=G_{F}\left(q_{i}\right)+\widetilde{\delta}\left(q_{i}\right), \quad \widetilde{\delta}\left(q_{i}\right)=2 \pi i \theta\left(q_{i}^{0}\right) \delta\left(q_{i}^{2}-m_{i}^{2}\right) .
$$

We define the advanced loop integral from the usual definition of the loop integral by replacing Feynman with advanced propagators:

$$
L_{A}^{(1)}\left(p_{1}, p_{2}, \ldots, p_{N}\right)=\int_{\ell_{1}} \prod_{i=1}^{N} G_{A}\left(q_{i}\right)
$$

Then by closing a contour on the lower $\ell_{1,0}-$ complex-plane and computing the integral with the residue method, we notice that:

$$
L_{A}^{(1)}\left(p_{1}, p_{2}, \ldots, p_{N}\right)=0
$$

since all the poles of the advanced propagators are in the positive half-plane. Replacing the advanced propagators with Feynman propagators, Eq.(2.6), we arrive at the relation:

$$
L^{(1)}\left(p_{1}, p_{2}, \ldots, p_{N}\right)=-\left[L_{1-\text { cut }}^{(1)}\left(p_{1}, p_{2}, \ldots, p_{N}\right)+\cdots+L_{N-\text { cut }}^{(1)}\left(p_{1}, p_{2}, \ldots, p_{N}\right)\right] .
$$

This equation is the FFT at one loop. It relates the one loop integral to multiple cut integrals. Each delta function $\widetilde{\delta}\left(q_{i}\right)$ in the multiple cut terms, replaces the corresponding Feynman propagator, by cutting the internal line with momentum $q_{i}$.

Rather than starting from $L_{A}^{(1)}$, we apply directly the residue theorem to the computation of $L^{(1)}$. Closing the integration contour at infinity in the direction of the negative imaginary axis, according to the Cauchy theorem, one picks up one pole from each of the $N$ Feynman propagators.

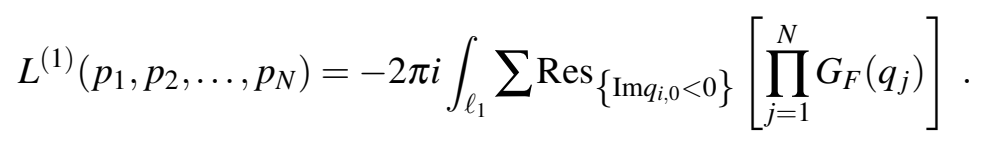

In Ref. [2], it is shown that the residue of these poles is given by:

$$
\operatorname{Res}_{\left\{\operatorname{Im} q_{i, 0}<0\right\}} \frac{1}{q_{i}^{2}-m_{i}^{2}+i 0}=\int d \ell_{1,0} \delta_{+}\left(q_{i}^{2}-m_{i}^{2}\right) .
$$

The value of the rest of the propagators at the residue, is shown to be [2] :

$$
\left[\prod_{j \neq i} G_{F}\left(q_{j}\right)\right]_{\left\{q_{i}^{2}-m_{i}^{2}=-i 0\right\}}=\prod_{j \neq i} \frac{1}{q_{j}^{2}-m_{j}^{2}-i 0 \eta\left(q_{j}-q_{i}\right)},
$$

where $\eta$ is a future-like vector:

$$
\eta_{\mu}=\left(\eta_{0}, \eta\right), \quad \eta_{0} \geq 0, \eta^{2}=\eta_{\mu} \eta^{\mu} \geq 0
$$

We finally obtain:

$$
L^{(1)}\left(p_{1}, p_{2}, \ldots, p_{N}\right)=-\sum_{i=1}^{N} \int_{\ell_{1}} \widetilde{\delta}\left(q_{i}\right) \prod_{j \neq i} G_{D}\left(q_{i} ; q_{j}\right)
$$

where

$$
G_{D}\left(q_{i} ; q_{j}\right)=\frac{1}{q_{j}^{2}-m_{j}^{2}-i 0 \eta\left(q_{j}-q_{i}\right)}
$$



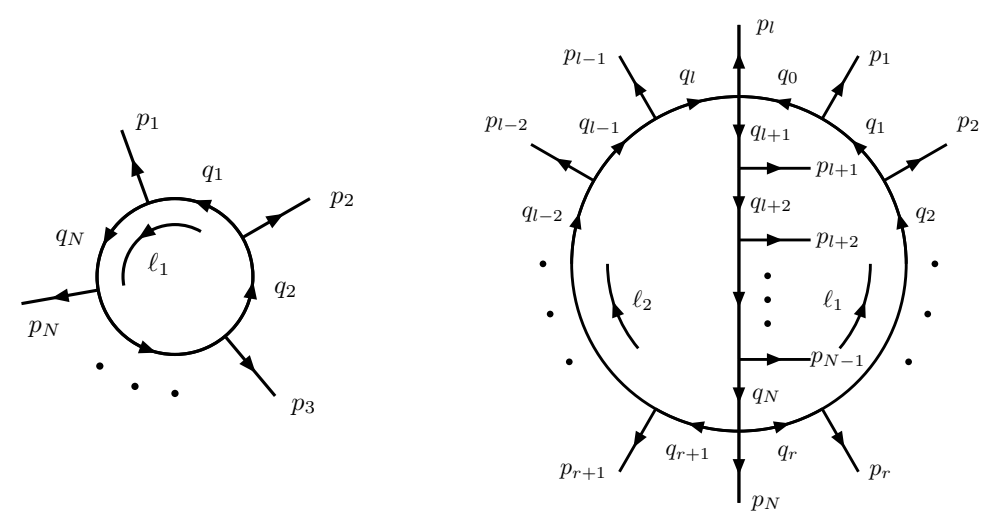

Figure 1: Momentum configuration of the one-loop and two-loop N-point scalar integral. Note, that in the two-loop case $p_{l}$ and $p_{N}$ can be equal to zero and hence the number of internal lines can differ from the number of external momenta.

is the dual propagator, which depends on the vector $\eta$. This is the duality theorem. We notice that since all the internal momenta depend on the same loop momentum, all dependence on it drops out and the $i 0$ prescription depends solely on the external momenta. This is an important trait and we will try to generalize it, in the next section, to more loops. The presence of the vector $\eta_{\mu}$ is a consequence of using the residue theorem and the fact that the residues at each of the poles are not Lorentz-invariant quantities. The Lorentz-invariance of the loop integral is recovered after summing over all the residues.

Finally we note that using the following relation between dual and Feynman propagators

$$
\widetilde{\boldsymbol{\delta}}\left(q_{i}\right) G_{D}\left(q_{i} ; q_{j}\right)=\widetilde{\boldsymbol{\delta}}\left(q_{i}\right)\left[G_{F}\left(q_{j}\right)+\tilde{\boldsymbol{\theta}}\left(q_{j}-q_{i}\right) \widetilde{\boldsymbol{\delta}}\left(q_{j}\right)\right],
$$

where $\tilde{\theta}(q)=\theta(\eta q)$, we can show that FFT and the duality theorem are equivalent [2].

\section{Duality theorem at two loops}

Our main goal is to write down the extension of the duality theorem in two- and higher loops keeping the dependence of the $i 0$ prescription at the dual propagator, on external momenta only. Also, we want to formulate it in such a way that we have the same number of cuts as the number of loops. At two loops the generic graph with $N$-legs is shown in Fig. 1. All momenta are taken outgoing and we now have two integration momenta $\ell_{1}, \ell_{2}$. To extend the duality theorem beyond one loop, it is useful to extend the definition of propagators of single momenta, to combinations of propagators of sets of internal momenta. To this end, let $\alpha_{k}$ be any set of loop momenta. We define the following functions of Feynman, advanced and dual propagators:

$$
\begin{aligned}
G_{F(A)}\left(\alpha_{k}\right) & =\prod_{i \in \alpha_{k}} G_{F(A)}\left(q_{i}\right) \\
G_{D}\left( \pm \alpha_{k}\right) & =\sum_{i \in \alpha_{k}} \widetilde{\delta}\left( \pm q_{i}\right) \prod_{\substack{j \in \alpha_{k} \\
j \neq i}} G_{D}\left( \pm q_{i} ; \pm q_{j}\right) .
\end{aligned}
$$

The minus sign in the definitions above, signifies a change in the flow of the momenta of the set. For a single momentum, $\alpha_{k}=\{i\}$ we define $G_{D}\left( \pm \alpha_{k}\right)=\widetilde{\delta}\left( \pm q_{i}\right)$. The relation between the three 
kind of propagators (see Eq.(2.6)), in the any-loop extended form, is now:

$$
G_{A}\left(\alpha_{k}\right)=G_{F}\left(\alpha_{k}\right)+G_{D}\left(\alpha_{k}\right) .
$$

With the notation described above, the one loop theorem, Eq.(2.14) can now be written in the compact form:

$$
L^{(1)}\left(p_{1}, p_{2}, \ldots, p_{N}\right)=\int_{\ell_{1}} G_{F}\left(\alpha_{1}\right)=-\int_{\ell_{1}} G_{D}\left(\alpha_{1}\right)
$$

When we have the union of several subsets of momenta, $\beta_{N} \equiv \alpha_{1} \cup \ldots \cup \alpha_{N}$ we can write the dual propagator function in terms of these subsets, by using the multiplicative properties of (3.1):

$$
G_{D}\left(\alpha_{1} \cup \alpha_{2} \cup \ldots \cup \alpha_{N}\right)=\sum_{\substack{\beta_{N}^{(1)} \cup \beta_{N}^{(2)} \\=\beta_{N}}} \prod_{i_{1} \in \beta_{N}^{(1)}} G_{D}\left(\alpha_{i_{1}}\right) \prod_{i_{2} \in \beta_{N}^{(2)}} G_{F}\left(\alpha_{i_{2}}\right)
$$

An example, that will be used shortly for the two-loop case, is when $N=2$, e.g. $\beta_{2}=\alpha_{1} \cup \alpha_{2}$ :

$$
G_{D}\left(\alpha_{1} \cup \alpha_{2}\right)=G_{D}\left(\alpha_{1}\right) G_{D}\left(\alpha_{2}\right)+G_{D}\left(\alpha_{1}\right) G_{F}\left(\alpha_{2}\right)+G_{F}\left(\alpha_{1}\right) G_{D}\left(\alpha_{2}\right)
$$

Let us now turn to the two loop case. Unlike the one-loop, the number of external momenta may be different from the number of internal momenta. The momenta of the internal lines are again denoted by $q_{i}$ and are explicitely given by

$$
q_{i}= \begin{cases}\ell_{1}+p_{1, i} & , i \in \alpha_{1} \\ \ell_{2}+p_{i, l-1} & , i \in \alpha_{2} \\ \ell_{1}+\ell_{2}+p_{i, l-1} & , i \in \alpha_{3},\end{cases}
$$

where $\alpha_{k}$, with $k=1,2,3$, are defined as the set of lines, propagators respectively, related to the momenta $q_{i}$, for the following ranges of $i$ :

$$
\alpha_{1}=\{0,1, \ldots, r\}, \quad \alpha_{2}=\{r+1, r+2, \ldots, l\}, \alpha_{3}=\{l+1, l+2, \ldots, N\} .
$$

The expression for the two-loop $N$-leg scalar integral is:

$$
L^{(2)}\left(p_{1}, p_{2}, \ldots, p_{N}\right)=\int_{\ell_{1}} \int_{\ell_{2}} G_{F}\left(\alpha_{1} \cup \alpha_{2} \cup \alpha_{3}\right) .
$$

Using the duality theorem sequentially, first for the loop momenta $\ell_{1}$ and the expression (3.5), we arrive at the dual representation of the two-loop scalar integral,

$$
L^{(2)}\left(p_{1}, p_{2}, \ldots, p_{N}\right)=-\int_{\ell_{1}} \int_{\ell_{2}}\left\{G_{D}\left(\alpha_{1}\right) G_{D}\left(\alpha_{3}\right)+G_{D}\left(\alpha_{1}\right) G_{F}\left(\alpha_{3}\right)+G_{F}\left(\alpha_{1}\right) G_{D}\left(\alpha_{3}\right)\right\} G_{F}\left(\alpha_{2}\right) .
$$

The first term of the integrand on the right-hand side of Eq.(3.9) is the product of two dual functions, and therefore already contains double cuts. We do not modify this term further. The second and third terms of (3.9) contain only single cuts and we thus apply the duality theorem again, i.e., use (3.3) for $\ell_{2}$. A subtlety arises at this point since due to our choice of momentum flow, $\alpha_{1}$ and $\alpha_{2}$ appearing in the third term of (3.9), flow in the opposite sense. Hence, in order to apply the duality theorem to the second loop, we have to reverse the momentum flow of one of these two 


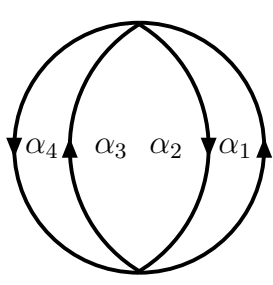

(a)

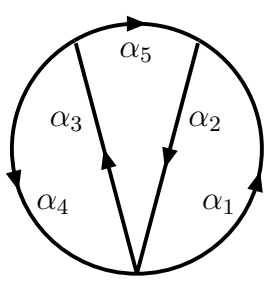

(b)

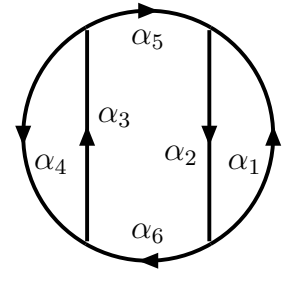

$(c)$

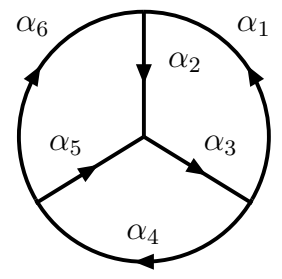

$(d)$

Figure 2: Master topologies of three-loop scalar integrals. Each internal line $\alpha_{i}$ can be dressed with an arbitrary number of external lines, which are not shown here.

loop lines. We choose to change the direction of $\alpha_{1}$, namely $q_{i} \rightarrow-q_{i}$ for $i \in \alpha_{1}$. Thus, applying (3.3) to the last two terms of (3.9) and expanding all parts in terms of the single loop lines of (3.7) leads to

$$
\begin{aligned}
& L^{(2)}\left(p_{1}, p_{2}, \ldots, p_{N}\right) \\
= & \int_{\ell_{1}} \int_{\ell_{2}}\left\{G_{D}\left(\alpha_{1}\right) G_{D}\left(\alpha_{2}\right) G_{F}\left(\alpha_{3}\right)+G_{D}\left(-\alpha_{1}\right) G_{F}\left(\alpha_{2}\right) G_{D}\left(\alpha_{3}\right)+G^{*}\left(\alpha_{1}\right) G_{D}\left(\alpha_{2}\right) G_{D}\left(\alpha_{3}\right)\right\},
\end{aligned}
$$

where

$$
G^{*}\left(\alpha_{k}\right) \equiv G_{F}\left(\alpha_{k}\right)+G_{D}\left(\alpha_{k}\right)+G_{D}\left(-\alpha_{k}\right) .
$$

In (3.10), the $i 0$ prescription of all the dual propagators depends on external momenta only. Through (3.11), however, (3.10) contains also triple cuts, given by the contributions with three $G_{D}\left(\alpha_{k}\right)$. The triple cuts are such that they split the two-loop diagram into two disconnected tree-level diagrams. By definition, however, the triple cuts are such that there is no more than one cut per loop line $\alpha_{k}$.

\section{Duality theorem at three loops and beyond}

Beyond two loops, the duality theorem applies in a similar manner. We expect to find at least the same number of cuts as the number of loops, and topology dependent disconnected tree diagrams built by cutting up to all the loop lines $\alpha_{k}$. For the case of three loops there are four master topologies, shown in Fig. 2. As an example, the basketball graph, Fig. 2a, is in terms of dual propagators:

$$
L_{\text {basket }}^{(3)}\left(p_{1}, p_{2}, \ldots, p_{N}\right)=\int_{\ell_{1}} \int_{\ell_{2}} \int_{\ell_{3}} G_{D}\left(\alpha_{1} \cup \alpha_{2}\right) G_{D}\left(\alpha_{3} \cup \alpha_{4}\right) .
$$

If we expand all existing dual functions in (4.1) in terms of dual functions of single loop lines by using (3.4) and apply the duality theorem to the third loop, we obtain

$$
\begin{aligned}
& L_{\text {basket }}^{(3)}\left(p_{1}, p_{2}, \ldots, p_{N}\right)=-\int_{\ell_{1}} \int_{\ell_{2}} \int_{\ell_{3}}\left\{G_{D}\left(\alpha_{2}, \alpha_{3},-\alpha_{4}\right) G_{F}\left(\alpha_{1}\right)+G_{D}\left(\alpha_{1}, \alpha_{3},-\alpha_{4}\right) G_{F}\left(\alpha_{2}\right)\right. \\
& \quad+G_{D}\left(-\alpha_{1}, \alpha_{2}, \alpha_{4}\right) G_{F}\left(\alpha_{3}\right)+G_{D}\left(-\alpha_{1}, \alpha_{2}, \alpha_{3}\right) G_{F}\left(\alpha_{4}\right) \\
& \left.\quad+G_{D}\left(-\alpha_{1}, \alpha_{2}, \alpha_{3}, \alpha_{4}\right)+G_{D}\left(\alpha_{1}, \alpha_{2}, \alpha_{3},-\alpha_{4}\right)+G_{D}\left(-\alpha_{1}, \alpha_{2}, \alpha_{3},-\alpha_{4}\right)\right\}
\end{aligned}
$$

where for brevity we use the notation: $G_{D}\left(\alpha_{1}, \ldots, \alpha_{N}\right)=\prod_{i=1}^{N} G_{D}\left(\alpha_{i}\right)$. For more details and the expressions for the rest of the topologies, we refer the reader to [8]. 


\section{Scattering Amplitudes}

The duality relation can be extended to compute scattering amplitudes. Since the application of duality affects only propagators, we can write down the analogue of Eq.(2.14) for amplitudes:

$$
\mathscr{A}^{(1-\text { loop })}=-\mathscr{A}^{(1-\text { loop })} \text {. }
$$

The expression $\tilde{\mathscr{A}}^{(1-\text { loop })}$ is obtained in the following manner: start from any diagram in $\mathscr{A}^{(1-\text { loop })}$ and consider all possible replacements of each Feynman propagator $G_{F}\left(q_{i}\right)$ with the cut propagator. The uncut propagators are replaced by the corresponding dual propagators. Eq.(5.1) establishes a correspondence between one-loop Feynman diagrams and the phase space integral of tree-level diagrams [2]. The duality relation for amplitudes is valid in any unitary and local field theory. In spontaneously broken gauge theories, it holds in the 't Hooft-Feynman gauge and in the unitary gauge. In unbroken gauge theories, the duality relation is valid in the 't Hooft-Feynman gauge, and in physical gauges where the gauge vector $n^{v}$ is orthogonal to the dual vector $\eta^{\mu}$, i.e., $n \cdot \eta=0$. This excludes gauges where $n^{v}$ is time-like. This choice of gauges at one-loop avoids the appearance of extra unphysical gauge poles, which in some gauges, moreover, become poles of higher order. At higher loop orders, the gauge poles will be absent with the same choice of gauges, and the duality relation can be extended straightforwardly to the amplitude level too.

For higher orders in pertubation theory, Eq.(5.1), generalizes to:

$$
\mathscr{A}^{(\mathrm{N}-\text { loop })}=(-1)^{N} \tilde{\mathscr{A}}^{(\mathrm{N}-\mathrm{loop})}
$$

where $\tilde{\mathscr{A}}^{\text {(N-loop) }}$ is the dual counterpart of the loop quantity $\mathscr{A}^{\text {(N-loop) }}$, which is obtained from the Feynman graphs in $\mathscr{A}^{(\mathrm{N}-\text { loop})}$ by sequentially applying on each loop the duality theorem. In [2], it was discussed how to evaluate the on-shell scattering amplitude, of $N$ external particles, from the tree-level forward scattering amplitude of $N+2$ particles, with two additional external legs of momenta $q$ and $-q$. The naive generalization to $N$-loops, requires the evaluation of tree-level scattering amplitudes with an even number of external legs, i.e. $q_{i}$ and $-q_{i}$, where $i$ runs from one to the number of loops (see also [9]). Care has to be taken though, when dealing with tadpoles and self energy insertions due to the appearence of kinematical singularities. These issues where already discussed in [2] and are left for future investigation.

\section{Conclusions}

We have rederived the tree-loop duality theorem at one-loop order, which was introduced in Ref. [2], in a way which is more suitable for extending it to higher loop orders. By applying iteratively the duality theorem, we have given explicit representations of the two- and three-loop scalar integrals. Dual representations of the loop integrals with complex dual prescription depending only on the external momenta can be obtained at the cost of introducing extra cuts, which break the loop integrals into disconnected diagrams. The maximal number of cuts agrees with the number of loop lines, and the cuts are such that there does not appear more than a single cut for each internal loop line. 


\section{Acknowledgments}

This work was supported by the Ministerio de Ciencia e Innovación under Grant No. FPA200760323, by CPAN (Grant No. CSD2007-00042), by the Generalitat Valenciana under Grant No. PROMETEO/2008/069, by the European Commission under contracts FLAVIAnet (MRTN-CT2006-035482) and HEPTOOLS (MRTN-CT-2006-035505), and by INFN-MICINN agreement under Grants No. ACI2009-1061 and FPA2008-03685-E.

\section{References}

[1] J. R. Andersen et al. [SM and NLO Multileg Working Group], "The SM and NLO multileg working group: Summary report," arXiv:1003.1241 [hep-ph].

[2] S. Catani, T. Gleisberg, F. Krauss, G. Rodrigo and J. C. Winter, "From loops to trees by-passing Feynman's theorem," JHEP 0809 (2008) 065 [arXiv:0804.3170 [hep-ph]].

[3] D. E. Soper, "QCD calculations by numerical integration," Phys. Rev. Lett. 81 (1998) 2638, "Techniques for QCD calculations by numerical integration," Phys. Rev. D 62 (2000) 014009; M. Kramer and D. E. Soper, "Next-to-leading order numerical calculations in Coulomb gauge," Phys. Rev. D 66 (2002) 054017.

[4] W. Kilian and T. Kleinschmidt, "Numerical Evaluation of Feynman Loop Integrals by Reduction to Tree Graphs," arXiv:0912.3495 [hep-ph].

[5] M. Moretti, F. Piccinini and A. D. Polosa, "A Fully Numerical Approach to One-Loop Amplitudes," arXiv:0802.4171 [hep-ph].

[6] R. P. Feynman, “Quantum theory of gravitation,” Acta Phys. Polon. 24 (1963) 697.

[7] R. P. Feynman, Closed Loop And Tree Diagrams, in Magic Without Magic, ed. J. R. Klauder, (Freeman, San Francisco, 1972), p. 355, in Selected papers of Richard Feynman, ed. L. M. Brown (World Scientific, Singapore, 2000) p. 867.

[8] I. Bierenbaum, S. Catani, P. Draggiotis and G. Rodrigo, "A Tree-Loop Duality Relation at Two Loops and Beyond," JHEP 10 (2010) 073.

[9] S. Caron-Huot, "Loops and trees," arXiv:1007.3224 [hep-ph]. 